\title{
Partitioning genetic effects due to embryo, cytoplasm and maternal parent for oil content in oilseed rape (Brassica napus L.)
}

\author{
Jian-Guo Wu, Chun-Hai Shi and Hai-Zhen Zhang \\ Department of Agronomy, College of Agriculture and Biotechnology, Zhejiang University, Hangzhou, China.
}

\begin{abstract}
Analysis of genetic main effects and genotype $x$ environment (GE) interaction effects on the oil content of oilseed rape (Brassica napus L.) was conducted by using a genetic model for the quantitative traits of seeds in diploid plants. The experiments were carried out over two years with 8 parents and a diallel mating design, which produced $F_{1}$ and $F_{2}$ generations. We found that the oil content of rape was simultaneously controlled by embryo genetic effect, cytoplasmic effects and maternal genetic effect as well as GE interaction effects, with the cytoplasmic and maternal effects playing the main role. The results indicate that selection of maternal plants for high oil content would be more efficient than selection based on single seeds. Since the GE interaction effects accounted for $73.69 \%$ of the total genetic effects and were more important than the genetic main effects it seems that selection might be influenced by environmental conditions. The estimate of narrow-sense heritability for oil content was $73.52 \%$ with the interaction heritability being larger than the general heritability, indicating that the early generations can be used for selection for high oil content. The prediction of genetic effects suggested that the parent cultivars Youcai 601 and Gaoyou 605 were better than the other cultivars for increasing oil content during the breeding of $B$. napus. The implications for the quantitative trait loci mapping of seed traits interfered by these three genetic systems is also discussed.
\end{abstract}

Key words: breeding selection, environmental interactions, heritability, QTL.

Received: May 24, 2005; Accepted: December 7, 2005.

\section{Introduction}

High oil content is always one of the most important aims in oilseed rape (Brassica napus L.) breeding programs, and genetic information on the quantitative traits involved will help breeders construct effective breeding procedures for developing new rape varieties with high oil content. The oil content of rape is an important quantitative trait influenced by environmental conditions (Jensen et al., 1996; Gutierrez Boem et al., 1996) but phenotypic variation in oil content is also mainly controlled by the genotype and genetic and environmental (GE) interaction effects.

It has been pointed out (Grami et al., 1977) that in summer rape broad-sense heritability for oil content is low $(0.263)$, as is narrow-sense heritability $(0.163)$, but for winter rape broad-sense heritability (0.812) and narrow-sense heritability (0.309) are relatively high (Han, 1990), with broad-sense heritability having been reported to be as high as 0.875 ( $\mathrm{Hu} 1987)$.

Seed genetic components, including additive and dominance effects, play an important role in the inheritance of oil content traits in oilseed rape (Brandle and McVetty,

Send correspondence to Chun-Hai Shi. Department of Agronomy, College of Agriculture and Biotechnology, Zhejiang University, Hangzhou 310029, China. E-mail: chhshi@ zju.edu.cn.
1988; Pai and Kumar, 1991; Han 1990) but this trait can also be significantly affected by GE interaction effects across environments (Brandle and McVetty, 1988; Pai and Kumar, 1991; Shafii et al., 1992). As in all plants, rape seeds represent a new generation which is different from the parental plants. However, the maternal plant provides nutrients for seed development, so not only embryo nuclear genes but genetic effects from the nuclear and cytoplasmic genes of the maternal plant could all be important components for the performance of rapeseed traits across environments.

Wang (1992) found that the $\mathrm{F}_{1} \mathrm{~s}$ oil content shows a significant positive correlation with the female parent (i.e. the maternal plant) and highly significant correlations between pollen parents (paternal plant genes) and $\mathrm{F}_{1} \mathrm{~s}$ oil content have also been were observed as have significant genetic effects for pollen genotype (Hom et al., 2003; Hom, 2004 and Hom et al., 2004). However, detailed genetic information on oil content inheritance in the three genetic systems is still lacking and there is a need to study genotype $\mathrm{x}$ environment (GE) interaction effects as well as the genetic main effects for rapeseed traits in three genetic systems (i.e. embryo, cytoplasm and maternal plants). 
Although it has been shown that the erucic acid content of rapeseeds is controlled by both genetic main effects and GE interaction effects (Shi et al., 2003), the genetic behavior from different oil content genetic systems has not been studied. In the present paper we used genetic models for quantitative traits of seeds in diploid plants to evaluate both the genetic main effects and GE interaction effects of embryo, cytoplasm and maternal plants and to estimate the heritabilities and breeding merit of parents used for improving the oil content of rape.

\section{Materials and Methods}

\section{Plant material, cultivation and experimental delineation}

The experiments were carried out from 1997 to 2000 . A diallel design was used in experiments with the following eight Brassica napus L parents: Youcai 601, Gaoyou 605, Huashuang 3, Yunyou 8, Zhongyou 821, Eyouchangjia, Zhong R-888 and Tower. Seeds of the parents were sown in 1997 and $F_{1}$ seeds obtained by crossing the eight parents using hand emasculation in the spring of 1998. Two environments were used in this study. For the first environment the parent and $F_{1} \mathrm{~S}$ seeds were sown on $7^{\text {th }}$ October 1998 and 31-day-old seedlings individually transplanted at a spacing of $35 \times 30 \mathrm{~cm}$ with 24 plants in each plot for parents and $F_{1}$. Before transplanting the seedlings any false $F_{1}$ seedlings were removed, producing a false seedling ratio of about $0.5 \%$ among the $\mathrm{F}_{1}$ generation. The experiment was laid out in a randomized block design with three field replications. Seed samples of parents and $\mathrm{F}_{2} \mathrm{~S}$ on the $\mathrm{F}_{1}$ plants were collected at maturity from eight plants in the middle part of each plot. Samples of $F_{1}$ seeds used for oil content analysis were obtained during the flowering season of 1999 using the isolated pollination method. The second environment experiment was started on $7^{\text {th }}$ October 1999 using the same procedure as described above.

\section{Oil content analysis}

The percentage oil content $(\mathrm{OC} \%, \mathrm{w} / \mathrm{w})$ of the rape seeds was analyzed using a NIRSystems model 5000 near-infrared reflectance spectroscope (NIRSystems, Inc., Silver Spring, MD, USA) according to the WinISI II manual instructions for routine analysis (FOSS NIRSystems/TECTOR, Infrasoft International, LLC.). In excess of $3 \mathrm{~g}$ of each intact sample were scanned in a $36 \mathrm{~mm}$ inner-diameter ring cup. The oil content calibration equation was developed using a standard normal variant + de-trending scatter correction and a 2, 4, 4, 1 mathematical treatment and a modified partial least square (MPLS) regression method (Wu et al., 2002a). When using such calibration parameters, the regression equations were used to estimate the oil content with a standard error of calibration (SEC) as low as $0.83 \%$ and a coefficient of determination (It is described by the $\mathrm{r}^{2}$ or RSQ) as high as 0.95 .

\section{Genetic analysis}

The genetic model used was an extension of the diploid plant seeds method for the analysis of cytoplasmic and maternal effects (Zhu and Weir, 1994) by including GE interaction effects for quantitative traits (Zhu, 1996). For a diallel mating design from a set of inbred lines, the generation mean $\left(y_{h i j k l}\right)$ of mating type $k$ from maternal line $i$ and paternal line $j$ in block $l$ of environment $h$ was partitioned as,

$$
y_{h i j k l}=\mu+E_{h}+G_{i j k}+G E_{h i j k}+B_{l(h)}+e h i j k l
$$

These six partitions were grouped into two parts, fixed values of $\mu$ (population mean) and $E_{h}$ (environmental effect), random values of $G_{i j k}$ (genetic main effect with components of embryo additive $(A)$ and dominance $(D)$ effects, cytoplasmic effect $(C)$, maternal additive $(A m)$ and dominance ( $D m$ ) effects), $G E_{h i j k}$ (GE interaction effect with components of embryo additive interaction $(A E)$ and dominance interaction $(D E)$ effects, cytoplasmic interaction effect $(C E)$, maternal additive interaction $(A m E)$ and dominance interaction $(D m E)$ effects), $B_{l(h)}$ (block effect), and $e_{h j k l}$ (residual effect).

The MINQUE (0/1) method (Zhu and Weir, 1994) was used to estimate variances and covariances. Phenotypic variance $\left(V_{P}\right)$ of the oil content trait was composed of the following genetic components,

$$
\begin{aligned}
& V_{P}=V_{G}+V_{G E}+2 C_{G o . G m}+2 C_{G o E . G m E}+V_{e}=\left(V_{A}+V_{D}+\right. \\
& \left.V_{C}+V_{A m}+V_{D m}\right)+\left(V_{A E}+V_{D E}+V_{C E}+V_{A m E}+V_{D m E}\right)+ \\
& 2\left(C_{A . A m}+C_{D . D m}\right)+2\left(C_{A E . A m E}+C_{D E . D m E}\right)+V_{e}
\end{aligned}
$$

where $V_{G}$ is the genetic main variance including $V_{A}$ (embryo additive variance), $V_{D}$ (embryo dominance variance), $V_{C}$ (cytoplasmic variance), $V_{A m}$ (maternal additive variance), $V_{D m}$ (maternal dominance variance) and $V_{G E}$ was GE interaction variance including $V_{A E}$ (embryo additive interaction variance), $V_{D E}$ (embryo dominance interaction variance), $V_{C E}$ (cytoplasmic interaction variance), $V_{A m E}$ (maternal additive interaction variance), $V_{D m E}$ (maternal dominance interaction variance). $C_{G o \cdot G m}$ (covariance between embryo and maternal main effect) could be divided into $C_{A \cdot A m}$ (covariance between embryo and maternal additive effect) and $C_{D \cdot D m}$ (covariance between embryo and maternal dominance effect), and $C_{G o E \cdot G m E}$ (covariance between embryo and maternal interaction effect) be partitioned into $C_{A E \cdot A m E}$ (covariance between embryo and maternal additive interaction effect), $C_{D E \cdot D m E}$ (covariance between embryo and maternal dominance interaction effect). $V_{e}$ was residual variance.

Since oil content performance might be simultaneously controlled by genetic main effects and GE interaction effects of genes, the estimated total narrow-sense oil content heritability $\left(h^{2}\right)$ was further partitioned into two 
parts of general heritability $\left(h_{G}^{2}\right)$ which had components of embryo general heritability $\left(h_{G o}^{2}=\left(V_{A}+C_{A . A m}\right) / V_{p}\right)$, cytoplasmic heritability $\left(h_{G c}^{2}=V_{C} / V_{P}\right)$, maternal general heritability $\left(h_{G m}^{2}=\left(V_{A m}+C_{A . A m}\right) / V_{p}\right)$ and interaction heritability $\left(h_{G E}^{2}\right)$ which had components of embryo interaction heritability $\left(h_{G O E}^{2}=\left(V_{A E}+C_{A E . A m E}\right) / V_{p}\right)$, cytoplasmic interaction heritability $\left(h_{G C E}^{2}=V_{C E} / V_{P}\right)$ and maternal interaction heritability $\left(h_{G m E}^{2}=\left(V_{A m E}+C_{A E . A m E}\right) / V_{p}\right)$.

The components of genetic main effects and GE interaction effects, including embryo additive effect $(A)$, cytoplasmic effect $(C)$, maternal additive effect $(\mathrm{Am})$, embryo additive interaction effect $(A E)$, cytoplasmic interaction effect $(C E)$ and maternal additive interaction effect $(A m E)$ were predicted by using an adjusted unbiased prediction method (Zhu, 1993; Zhu and Weir, 1996). Sampling generation means of entries, the standard errors of estimated variances, heritabilities and predicted genetic effects were derived using the Jackknife method (Miller, 1974; Zhu and Weir, 1996).

\section{Results}

\section{Phenotypic variant of the generations}

There was great variation in the mean and range of the oil content of the parent plants and the $\mathrm{F}_{1}$ and $\mathrm{F}_{2}$ generations (Figure 1). The oil content of the parent plants ranged from 34.1 to $43.8 \%$ in the 1999 trials and 30.5 to $40.3 \%$ in the 2000 trials, these values being suitable for genetic crossing designs. A similar situation occurred with the $F_{1}$ and $F_{2}$ generations and since the ranges and the means of the each generation fluctuated between the two years (Figure 1), the performance of the generations in terms of oil content could be affected by the genotype and influenced by environmental conditions. The means of $F_{2}$ generation in both years were larger than those of their parents their parents in Figure 1 , indicating that high oil content of $F_{2}$ seeds could be improved through the utilization of hybrid vigor rape from hybrid seed $\left(\mathrm{F}_{1}\right)$.

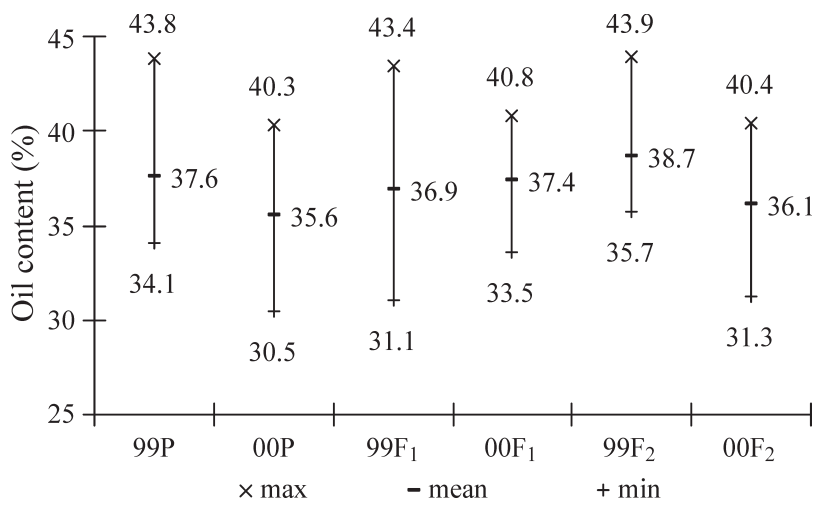

Figure 1 - Phenotypic distribution of generations for rape oil content. Parents $=99 \mathrm{P}(1999)$ and 00P (2000), $\mathrm{F}_{1}=99 \mathrm{~F}_{1}(1999)$ and $00 \mathrm{~F}_{1}(2000)$; $\mathrm{F}_{2}=99 \mathrm{~F}_{2}(1999)$ and $00 \mathrm{~F}_{2}(2000)$.

\section{Estimation of components for genetic main variance and $\mathrm{GE}$ interaction variance}

The oil content variance components of rape seeds are shown in Figure 2. The genetic main variances and GE interaction variances for embryo, cytoplasm and maternal effects were significant, except for $V_{D}$ and $V_{D m}$. Since the proportion of $V_{G E}$ to total genetic variance $(73.69 \%)$ was larger than that of $V_{G}(26.31 \%)$ for oil content, environments could mainly affect the expression of genes controlling this trait. It was suggested that GE interaction effects could not be neglected in high oil content breeding of rapeseed. For genetic main effects, there existed significant components of embryo additive variance $\left(V_{A}\right)$, cytoplasmic variance $\left(V_{C}\right)$ and maternal additive variance $\left(V_{A m}\right)$, whereas embryo dominant variance $\left(V_{D}\right)$ and maternal dominant variance $\left(V_{D m}\right)$ were not found. The genetic main effects for oil content were mostly contributed by maternal effects because $V_{A m}$ was larger than $V_{C}$ and $V_{A}$. Since maternal interaction effects, including additive $(33.86 \%)$ and dominant $(22.43 \%)$ interaction effects, were accounted for $56.29 \%$ of total GE interaction variances, the maternal interaction effects had played a much more important role for oil content than the other interaction components among GE interaction effects. It was revealed, by the larger additive and cytoplasm main variances $\left(\left(V_{A}+V_{C}+V_{A m}\right) / V_{G}=\right.$ $100.00 \%)$, and additive and cytoplasm interaction variances $\left(\left(V_{A E}+V_{C E}+V_{A m E}\right) / V_{G E}=69.97 \%\right)$, that additive and cytoplasmic effects were more important than other genetic effects. Thus breeding aim of high oil content could be achieved by selection in early generations.

Although the residual variances $\left(V_{e}\right)$ were significant and the performance of oil content of rapeseed could be influenced by sampling errors such as false hybrid seed in $F_{1}$ generation, it was concluded that oil content was mainly controlled by genetic main effects and GE interaction effects from different genetic systems, because of the small estimated residual variance. Since $\mathrm{C}_{A \cdot A m}, \mathrm{C}_{D \cdot D m}, \mathrm{C}_{A E \cdot A m E}$ and $\mathrm{C}_{D E \cdot D m E}$ were all not significant in the present experiment (not shown in Figure 2), no significant relationship

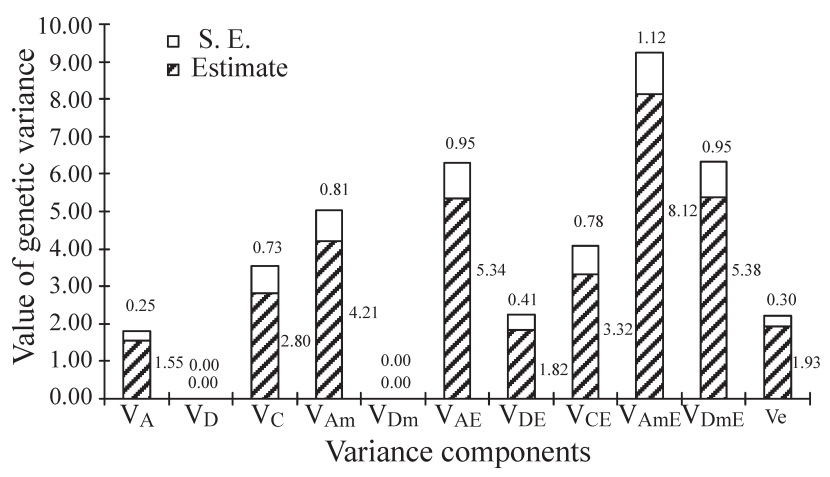

Figure 2 - Estimates of genetic variance components for rape oil content. Values on the upper and right side of the columns are standard error (SE) and variance component estimates. All estimates except for $V_{D}$ and $V_{D m}$ were significant at $\mathrm{p}=0.01$. 
between the embryo and maternal main effects or between embryo and maternal interaction effects were found.

\section{Estimation of heritability components}

The estimate of the total narrow-sense heritability $\left(h^{2}\right)$ was $73.52 \%$ for oil content (Figure 3), while the interaction heritability $\left(h_{G E}^{2}\right)(48.68 \%)$ for oil content was found to be larger than the general heritability $\left(h_{G}^{2}\right)(24.84 \%)$. Therefore, selection efficiency of seeds could be influenced by the environment conditions. The total of maternal and cytoplasm heritabilities including maternal general heritability $\left(h_{G m}^{2}\right)$, cytoplasmic general heritability $\left(h_{G c}^{2}\right)$, maternal interaction heritability $\left(h_{G m E}^{2}\right)$ and cytoplasm interaction heritability $\left(h_{G C E}^{2}\right)$ was found as high as $53.54 \%$, while the total embryo heritability including embryo general heritability $\left(h_{G o}^{2}\right)$ and embryo interaction heritability $\left(h_{G o E}^{2}\right)$ was smaller one with $19.98 \%$. Thus, improving oil content of rape seeds would be more efficient when selection was based on maternal plants than on early generations of seeds. The above analysis shows that oil content heritability was so complicated that it was composed of six heritability components all of which were significant, although maternal heritability was the most important.

\section{Analysis of genetic effects for parents}

Oilseed rape breeders are mainly interested in the genetic merits of parents because they need better parents in the breeding schedule. The genetic effect of oilseed rape parents was successfully divided between the embryo additive effect, the cytoplasmic effect, the maternal additive effect and their corresponding environmental interaction effects. Our results showed that the genetic main effects $(A$, $C$ and $A m)$ as well as GE interaction effects $(A E, C E$ and $A m E$ ) of parents could affect oil content performance in offspring and detectable differences were found for the genetic main effects and GE interaction effects among parents (Table 1). In 1999 the total genetic effects of oil content for

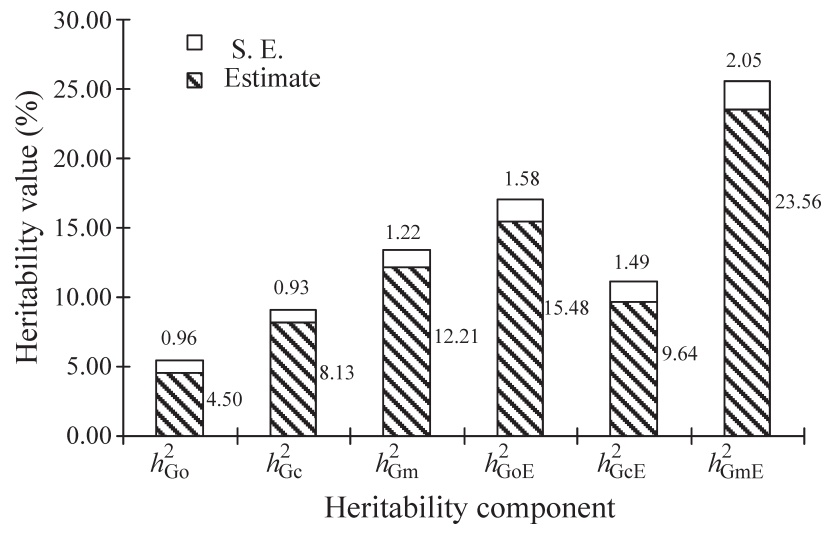

Figure 3 - Estimates of percentage oil content heritability components for oilseed rape. Values on the upper and right side of the columns are standard error (SE) and variance component estimates. All estimates were significant at $\mathrm{p}=0.01$.

the Youcai 601 parent was $1.19 \%$ and $4.37 \%$ for the Gaoyou 605 parent $\left(G_{t} \mathrm{I}=A+A E \mathrm{I}+C+C E \mathrm{I}+A m+A m E\right.$ I) but in 2000 it was $1.56 \%$ for the Youcai 601 parent and $5.47 \%$ for the Gaoyou 605 parent $\left(G_{t} \mathrm{II}=A+A E \mathrm{II}+C+\right.$ $C E \mathrm{II}+A m+A m E$ II). Therefore, they could be used as parents for increasing oil content in rape quality breeding because of their stability for oil content performance in different environments (years). The oil content of the progenies of some parents was low, e.g. Yunyou 8 (Oil contents were negative $3.20 \%$ for 1999 and $1.96 \%$ for 2000 ), Zhongyou 821 (negative $0.99 \%$ for 1999 and $0.05 \%$ for 2000) and Zhong R-888 (negative 2.93\% for 1999 and $1.38 \%$ for 2000 ) and these varieties do not appear to be suitable parents for improving the oil content of progenies in high oil content breeding programs.

\section{Discussion}

Oil is always the main product from rape varieties and it is one of main breeding aims to increase it. There is wide

Table 1 - Predication of genetic main effects and GE interaction effects for oil content (\%) of rape seeds.

\begin{tabular}{|c|c|c|c|c|c|c|c|c|c|c|c|}
\hline \multirow{2}{*}{$\begin{array}{l}\text { Parent } \\
\text { cultivar }\end{array}$} & \multicolumn{3}{|c|}{ Embryo additive effect } & \multicolumn{3}{|c|}{ Cytoplasmic effect } & \multicolumn{3}{|c|}{ Maternal additive effect } & \multirow{2}{*}{$\begin{array}{c}\mathrm{G}_{t} \mathrm{I} \\
1999\end{array}$} & \multirow{2}{*}{$\begin{array}{l}\mathrm{G}_{t} \mathrm{II} \\
2000\end{array}$} \\
\hline & A & AE I & AE II & $\mathrm{C}$ & CE I & CE II & $\mathrm{Am}$ & AmE I & AmE II & & \\
\hline Youcai 601 & 0.16 & $3.29 * * *$ & $-2.60 * * *$ & 1.55 & 1.73 & -0.43 & -0.78 & $-4.77 * * *$ & $3.66 * * *$ & 1.19 & 1.56 \\
\hline Gaoyou 605 & -0.11 & $-2.68 * *$ & $2.64 * *$ & $3.26^{*}$ & 3.12 & 0.04 & -0.41 & 1.19 & 0.04 & 4.37 & 5.47 \\
\hline Huashuang 3 & -0.04 & $-2.22 * * *$ & 0.40 & -0.28 & -0.65 & 0.41 & 0.12 & $1.38 *$ & -0.30 & -1.68 & 0.31 \\
\hline Yunyou 8 & -0.02 & $-2.67 * * *$ & $1.71 * * *$ & $-1.91 * *$ & -0.38 & -1.13 & 0.31 & $1.47 * * *$ & -0.92 & -3.20 & -1.96 \\
\hline Zhongyou 821 & -0.04 & $1.90 * * *$ & $-2.05 * * *$ & -0.33 & $-2.09 * *$ & $1.93 *$ & 0.14 & -0.58 & 0.30 & -0.99 & -0.05 \\
\hline Eyouchangjia & 0.28 & $6.57 * *$ & $-2.53 * *$ & -1.72 & -1.86 & 0.05 & 0.21 & $-2.80 * * *$ & 1.18 & 0.67 & -2.53 \\
\hline Zhong R-888 & -0.18 & $-2.62 * *$ & 0.85 & -0.74 & -1.32 & 0.92 & 0.25 & $1.68 * *$ & $-2.48 * * *$ & -2.93 & -1.38 \\
\hline Tower & -0.05 & $-1.57 * * *$ & $1.57 * * *$ & 0.17 & 1.44 & -1.80 & 0.15 & $2.42 * * *$ & $-1.47 * *$ & 2.56 & -1.44 \\
\hline
\end{tabular}

$*$ = significant at $0.10 ; * *$ significant at 0.05 ; significant at $0.01^{* * *} ; \mathrm{A}=$ embryo additive effect; $\mathrm{AE}=$ embryo additive interaction effect; $\mathrm{C}=$ cytoplasmic effect; $\mathrm{CE}=$ cytoplasmic interaction effect; $\mathrm{Am}=$ maternal additive effect; $\mathrm{AmE}=$ maternal additive interaction effect; $G_{t} \mathrm{I}=A+A E \mathrm{I}+C+C E \mathrm{I}+A m+$ $A m E \mathrm{I} ; G_{t} \mathrm{II}=A+A E \mathrm{II}+C+C E \mathrm{II}+A m+A m E \mathrm{II}$. 
variability in the oil content of rape germplasm, which provides the opportunity for improving the quality of rape varieties via breeding (Bhardwaj and Hamama, 2000). In order to increase the efficiency of rape breeding, breeders are now paying much more attention to elucidating the genetic mechanism, which control for oil content in rape seeds.

Most genetic research on the oil content of oilseed rape has focused on the genetic information contained in nuclear genes and has found that the oil content performance is controlled by additive and dominance effects as well as their environment effects (Brandle and McVetty, 1988; Pai and Kumar, 1991), although some research has also found that cytoplasmic or maternal effects could also influence the oil content performance (Wang, 1992). However, there was no information appears to be available on how the oil content of rape can be simultaneously affected by the genetic main effects from the different genetic systems (i.e. diploid embryo nuclear genes, cytoplasmic genes and diploid maternal plant nuclear genes) and genotype $\mathrm{x}$ environment (GE) interaction effects. If the seed traits were controlled by the genes of the three genetic systems at the same time then the genetic estimations for seed traits obtained from only one genetic system without excluding the influences of other genetic systems would be biased and might mislead breeders. Recently, quantitative trait locus (QTL) mapping has been conducted on oilseed rape to dissect the effects of individual loci for seed traits in terms of rape-oil yield (Tanhuanpää et al., 1996; Thormann et al., 1996; Zhao et al., 2005). QTL analysis in which the molecular marker near the available locus can be screened is important in molecular breeding strategies such marker associated selection (MAS) used to screen the target materials precisely and improve selection efficiency for quantitative traits (Tanhuanpää et al., 1996; Somers et al., 1998; Gupta et al., 2004). So far, QTL mapping for seed traits has been based on the maternal plant because of the QTL method used to map agriculturally plant traits. Due to the nutrient and physiological substances related to the three genetic systems operating during the growth and development of seeds, QTL mapping for seed traits should consider not only maternal effects but also interference embryo and cytoplasm genetic effects.

In this paper we have successfully produced genetic information on the embryo nuclear genes, cytoplasmic genes and diploid maternal plant nuclear genes and their GE interaction effects in respect of the oil content of dicotyledon rape. We found that although oil content performance was affected both by genetic main effects from nuclear genes and GE interaction effects, in which all the individual components could be even more important than those in the main effects in terms of oil content performance.

Our genetic analysis found that the oil content of rapeseed was mainly controlled by maternal and cytoplasm genetic effects with high respective heritabilities, suggesting that improving oil content of rapeseed would be more efficient when selection was based on maternal plants than on seeds from early generations. It thus seems that selection methods might be designed based on maternal single plant performance because the single seed selection method for oil content had low efficiency for rapeseed breeding. The two problems, large amounts of sample and the small size of each sample tested, should be resolved when screening single plants in breeding programs. Also, non-invasive analysis techniques such as nuclear-magnetic resonance (NMR) and near-infrared reflectance spectroscopy (NIRS) techniques could be applied to screen single plant seeds in early generation in breeding programs (Brühl and Matthäus, 1999; Wu et al., 2002a, b). When using the NIRS calibration equation to select single seeds, Hom (2004) found that the selection of single seeds has certain responses to selection for oil.

It is very important for breeders worldwide to select suitable parents in rapeseed pure-line breeding programs for improving breeding efficiency. Good parent plants which have the best genetic effects and are suited to different environments for rapeseed quality breeding may be able to be selected by predicting breeding values. Additionally, the approach outlined in this paper appears to give the most detailed genetic information on embryo, cytoplasmic and maternal additive effects as well as their environments interactions for the rape oil content trait and allows breeders to make this work. In this study we found that all parents possessed the appropriate genetic components and that some parents such as Youcai 601 and Gaoyou 605 had better breeding values, which could increase the oil content of rape in different environments (years), and that these two parents perform consistently across environments and could be used as parents in rapeseed quality breeding for increasing oil content.

\section{Acknowledgments}

The project was financially supported by the Foundation for University Key Teacher by the Ministry of Education of China and by the 151 Program for the Talents of Zhejiang Province. The authors also thank Prof. Zhu for providing the analysis software. We thank Prof. Jian-Shen $\mathrm{Wu}$ and Prof. Wei-Jun Zhou for providing the rapeseed varieties used as parents in the present experiment.

\section{References}

Bhardwaj HL and Hamama AA (2000) Oil, erucic acid, and glucosinolate contents in winter hardy rapeseed germplasms. Industry Crop Prod 12:33-38.

Brandle JE and McVetty PBE (1988) Effects of inbreeding and estimates of additive genetic variance within seven summer oilseed rape cultivars. Genome 32:115-119.

Brühl L and Matthäus B (1999) Extraction of oilseeds by SFE- a comparison with other methods for the determination of the oil content. Fresenius J Analyt Chem 364:631-634. 
Grami B, Baker RJ and Stefansson BR (1977) Genetics of protein and oil content in summer rape, heritability, number of effective factors and correlations. Canad J Plant Sci 57:937943.

Gupta V, Mukhopadhyay A, Arumugam N, Sodhi YS, Pental D and Pradhan AK (2004) Molecular tagging of erucic acid trait in oilseed mustard (Brassica juncea) by QTL mapping and single nucleotide polymorphisms in FAE1 gene. Theor Appl Genet 108:743-749.

Gutierrez Boem FH, Lavado RS and Porcelli CA (1996) Note on the effects of winter and spring waterlogging on growth, chemical composition and yield of rapeseed. Field Crops Res 47:175-179.

Han JX (1990) Genetic analysis on oil content in rapeseed (Brassica napus L). Oil Crop China 2:1-6.

Hom NH (2004) Pollen Genotype Effects on Seed Quality and Selection of Single Seeds by Near-Infrared Reflectance Spectroscopy (NIRS) in Winter Oilseed Rape. Diss GeorgAugust-University, Göttingen, 36 pp.

Hom NH, Girke A, Möllers C and Becker HC (2003) Influence of pollen genotype on rapeseed quality. Proc of the 11th International Rapeseed Congress Copenhagen, Denmark, v. 1, pp 266-269.

Hom NH, Girke A, Möllers C and Becker HC (2004) Pollen genotype effects on seed quality traits in winter oilseed rape. Vort Pflanzenzücht 64:95-97.

Hu ZL (1987) Genetic anlysis on several quality traits in rapeseed (Brssica napus L). Oil Crop China 1:19-22.

Jensen CR, Mogensen VO, Mortensen G, Fieldsend JK, Milford GFJ, Andersen MN and Thage JH (1996) Seed glusosinolate, oil and protein contents of field-grown rape (Brassica napus L) affected by soil drying and evaporative demand. Field Crops Res 47:93-105.

Miller RG (1974) The Jackknife, a review. Biometrika 61:1-15.

Pai R and Kumar P (1991) Estimates of gene effects of oil content under normal and late sowing in mustard (Brassica juncea). Indian J Agron Sci 61:918-921.

Shafii B, Mahler KA, Price WJ and Auld DL (1992) Genotype x environment interaction effects on winter rapeseed yield and oil content. Crop Sci 32:922-927.
Shi CH, Zhang HZ, Wu JG, Li CT and Ren YL (2003) Genetic and genotype $\mathrm{x}$ environment interaction effects analysis for erucic acid content in rapeseed (Brassica napus L). Euphytica 130:249-254.

Somers DJ, Friesen KRD and Rakow G (1998) Identification of molecular markers associated with linoleic acid desaturation in Brassica napus. Theoretical and Applied Genetics 96:897-903.

Tanhuanpää PK, Vilkki JP and Vilkki HJ (1996) Mapping of a QTL for oleic acid concentration in spring turnip rape (Brassica rapa ssp oleifera). Theoretical and Applied Genetics 92:952-956.

Thormann CE, Romero J, Mantet J and Osborn TC (1996) Mapping loci controlling the concentrations of erucic and linolenic acids in seed oil of Brassica napus L. Theoretical and Applied Genetics 93:282-286.

Wang TQ (1992) The heredity of oil content and its heterosis in rapeseed. Journal of Guizhou Agriculture Science 6:37-40.

Wu JG, Shi CH, Zhang HZ and Fan LJ (2002a) Study on analysis of oil content of intact rapeseed with few sample size by near infrared reflectance spectroscopy. Acta Agronomica Sinica 28:421-425.

Wu JG, Shi CH and Fan LJ (2002b) Calibration optimization for analysing erucic acid and glucosinolate content of rapeseed by near infrared reflectance spectroscopy (NIRS). Journal of Chinese Cereal Oils Association 17:59-62.

Zhao JY, Becker HC, Zhang DQ, Zhang YF and Ecke W (2005) Oil content in a European $\mathrm{x}$ Chinese rapeseed population: QTL with additive and epistatic effects and their genotype-environment interactions. Crop Science 45:51-59.

Zhu J and Weir BS (1994) Analysis of cytoplasmic and maternal effects. I. A genetic model for diploid plant seeds and animals. Theoretical and Applied Genetics 89:153-159.

Zhu J and Weir BS (1996) Diallel analysis for sex-linked and maternal effects. Theoretical and Applied Genetics 92:1-9.

Zhu J (1993) Methods of predicting genotype value and heterosis for offspring of hybrids. Chinese Journal of Biomathematics 8:32-44

Zhu J (1996) Analytic methods for seed models with genotype $x$ environment interactions. Acta Genetica Sinca 23:56-68. Associate Editor: Márcio de Castro Silva Filho 